\title{
BMJ Open Effectiveness and implementation of enhanced recovery after surgery programmes: a rapid evidence synthesis
}

\author{
Fiona Paton, ${ }^{1}$ Duncan Chambers, ${ }^{1}$ Paul Wilson, ${ }^{1}$ Alison Eastwood, ${ }^{1}$ Dawn Craig, ${ }^{1}$ \\ Dave Fox, ${ }^{1}$ David Jayne, ${ }^{2}$ Erika McGinnes ${ }^{2}$
}

To cite: Paton F,

Chambers D, Wilson $\mathrm{P}$, et al.

Effectiveness and

implementation of enhanced

recovery after surgery

programmes: a rapid

evidence synthesis. BMJ

Open 2014;4:e005015.

doi:10.1136/bmjopen-2014005015

- Prepublication history and additional material is available. To view please visit the journal (http://dx.doi.org/ 10.1136/bmjopen-2014005015).

Received 7 February 2014 Revised 1 July 2014 Accepted 4 July 2014

\section{SLinked}

- http://dx.doi.org/10.1136/ bmjopen-2014-005014

\section{CrossMark}

${ }^{1}$ Centre for Reviews and Dissemination, University of York, York, UK

${ }^{2}$ Leeds Teaching Hospitals NHS Trust, Leeds, UK

Correspondence to

Fiona Paton;

fiona.paton@york.ac.uk

\section{ABSTRACT}

Objectives: To assess the evidence on the impact of enhanced recovery programmes for patients undergoing elective surgery in acute hospital settings in the UK

Design: Rapid evidence synthesis. Eight databases were searched from 1990 to March 2013 without language restrictions. Relevant reports and guidelines, websites and reference lists of retrieved articles were scanned to identify additional studies. Systematic reviews, RCTs not included in the systematic reviews, economic evaluations and UK NHS cost analysis, implementation case studies and surveys of patient experience in a UK setting were eligible for inclusion. Primary and secondary outcome measures: We assessed the impact of enhanced recovery programmes on health or cost-related outcomes, and assessed implementation case studies and patient experience in UK settings. Studies were quality assessed where appropriate using the Centre for Reviews and Dissemination Database of Abstracts of Reviews of Effects critical appraisal process.

Results: 17 systematic reviews and 12 additional RCTs were included. Ten relevant economic evaluations were included. No cost analysis studies were identified. Most of the evidence focused on colorectal surgery. 14 innovation case studies and 15 implementation case studies undertaken in National Health Service settings described factors critical to the success of an enhanced recovery programme.

Evidence for colorectal surgery suggests that enhanced recovery programmes may reduce hospital stays by $0.5-3.5$ days compared with conventional care. There were no significant differences in reported readmission rates. Other surgical specialties showed greater variation in reductions in length of stay reflecting the limited evidence identified. Findings relating to other outcomes were hampered by a lack of robust evidence and poor reporting.

Conclusions: There is consistent, albeit limited, evidence that enhanced recovery programmes can reduce length of patient hospital stay without increasing readmission rates. The extent to which managers and clinicians considering implementing enhanced recovery programmes in UK settings can realise savings will depend on length of stay achieved under their existing care pathway.
Strengths and limitations of this study

- Enhanced recovery programmes have been adopted with enthusiasm by the NHS as a means to achieving productivity gains and cost savings. This evolution makes combining studies over different periods and interpreting results of earlier studies in relation to the current context more difficult.

- The evidence base to support such widespread implementation suggests possible benefits in terms of reduced length of hospital stay, fewer postoperative complications, reduced readmissions and improved patient outcomes.

- Although there is a reasonable volume of evidence evaluating enhanced recovery programmes in colorectal surgery, robust evidence is sparse. Optimal care is certainly the right thing to do, but the evidence does not identify which enhanced recovery programme elements and combinations of elements are most effective.

- Findings relating to other outcomes, costs of enhanced recovery programmes, experience in using the programmes, and patient experience were limited by generally poor quality evidence and poor reporting. As such, conclusions on which combinations provide greatest gains and how best to implement them cannot be made.

\section{INTRODUCTION}

The National Health Service (NHS) faces severe funding constraints now and in the medium term. The forecast reduction in resources provides an enormous challenge to NHS organisations and staff. Service redesign can save money and improve quality but much depends on how care is co-ordinated and the way services are implemented in a local setting. ${ }^{12}$ NHS decisionmakers need to consider not only the effectiveness and costeffectiveness of any initiative but also efficient implementation. Enhanced recovery programmes (also known as ERAS, fast track, multimodal, rapid or accelerated recovery programmes) seek to deliver an optimal 
pathway (covering the preoperative, intraoperative and postoperative periods) that is focused on optimal recovery and discharge for patients. The approach was pioneered in Denmark in the late 1990s for patients undergoing colorectal surgery ${ }^{3}$ and is now spreading to other surgical pathways such as orthopaedic, urology and gynaecology.

Enhanced recovery programmes have been delivered in the UK NHS since the early 2000s. Implementation has to date been variable despite the support of the Department of Health and more recently the Royal Colleges. In 2011, 14 innovation sites were established as part of the Enhanced Recovery Partnership Programme. These sites acted as pathfinders for implementation; some sites were self-selecting and others were encouraged to join. The aim was to raise the profile, promote the benefits and inform the uptake of enhanced recovery for elective surgical care across the NHS. These sites had little or no experience in enhanced recovery pathways. It is likely that this variation seen across these sites reflects the complexity of enhanced recovery programmes themselves and issues around implementing change in established surgical pathways. Differences in programme implementation may also reflect differences between surgical specialities. Set against the benefits of enhanced recovery programmes are concerns that discharging patients too soon after surgery could increase complications and readmissions, thereby worsening patient experience and potentially health outcomes, and increasing pressure on primary and/or secondary healthcare services.

Before embarking on adoption of an enhanced recovery programme, NHS managers and clinicians need to be fully aware of the strength of the underlying evidence. They need to have a clear understanding of how best to implement such programmes and the likely implications for service delivery within finite budgets and considering the need for equity of access. The aim of this project was to conduct a rapid synthesis of the evidence on the clinical and cost-effectiveness of enhanced recovery programmes, and the implementation, delivery and impact of such programmes in secondary care settings in the UK.

\section{METHODS}

Eight databases, including DARE, NHS EED and MEDLINE were searched to from 1990 to March 2013 without language restrictions. The PROSPERO database was searched to identify ongoing systematic reviews. Relevant reports and guidelines were screened for further studies. Reference lists of retrieved articles, reviews and evaluations were scanned, and relevant individuals contacted for additional evidence.

Systematic reviews, RCTs not included in the systematic reviews, economic evaluations and UK NHS cost analysis studies were included if they evaluated the impact of enhanced recovery programmes (encompassing different combinations of the main preoperative, intraoperative and postoperative pathway elements described by the Enhanced Recovery Partnership Programme $)^{4}$ on health-related or cost-related outcomes. Eligible studies included patients undergoing elective surgery in an acute hospital in the UK NHS or a comparable healthcare system. Comparators were only relevant to clinical and cost-effectiveness evaluations, and included conventional (usual/standard) care without a structured multimodal enhanced recovery patient pathway (as defined in the included studies). Case studies, impact assessments and surveys of patient experience that documented the experience of implementing enhanced recovery in a UK setting were also eligible.

Quality assessment of systematic reviews, RCTs and economic evaluations was based on existing CRD critical appraisal methods (http://www.crd.york.ac.uk/crdweb/ HomePage.asp; CRD, 2009). Cost analysis studies, studies of patient experience and case studies of implementation were not formally quality assessed.

All stages of the review process were performed by one researcher and checked by a second. Disagreements between reviewers were resolved by discussion or by recourse to a third reviewer where necessary.

The type and range of evidence precluded metaanalysis and we therefore performed a narrative synthesis, differentiating clinical outcomes (eg, mobilisation, mortality and morbidity and length of hospital stay), patientreported outcomes (eg, patient experience and satisfaction), resource use in secondary care (eg, workforce utilisation and costs) and implementation case studies.

\section{RESULTS}

Seventeen systematic reviews ${ }^{5-21}$ and 12 additional $\mathrm{RCTs}^{22-34}$ were included in the evidence on clinical effectiveness (see figure 1: flow diagram). The quality of the systematic reviews varied and the additional RCTs were considered to be at high risk of bias (see tables 1 and 2). One RCT was a four arm trial; this was the only multicentre trial, the remaining trials were small, single centre trials. ${ }^{35}$ We included 15 case studies of implementation of ERAS in NHS settings, and evaluations of the 14 Enhanced Recovery Partnership Programme innovation sites. In addition, 10 relevant economic evaluations were also included (summary evidence tables are available on request from the review authors). Most of the evidence focused on colorectal surgery.

Where reviews reported the number of included patients, sample sizes ranged between 99 and 5747 patients in the ERAS group and between 99 and 1062 in comparator groups. Most individual RCTs analysed fewer than 100 patients (range 44-597 patients). Where indications for surgery were reported in systematic reviews and individual RCTs, most trials were in patients with cancer. Where reported, patients were adults within 
Figure 1 Study flow diagram.

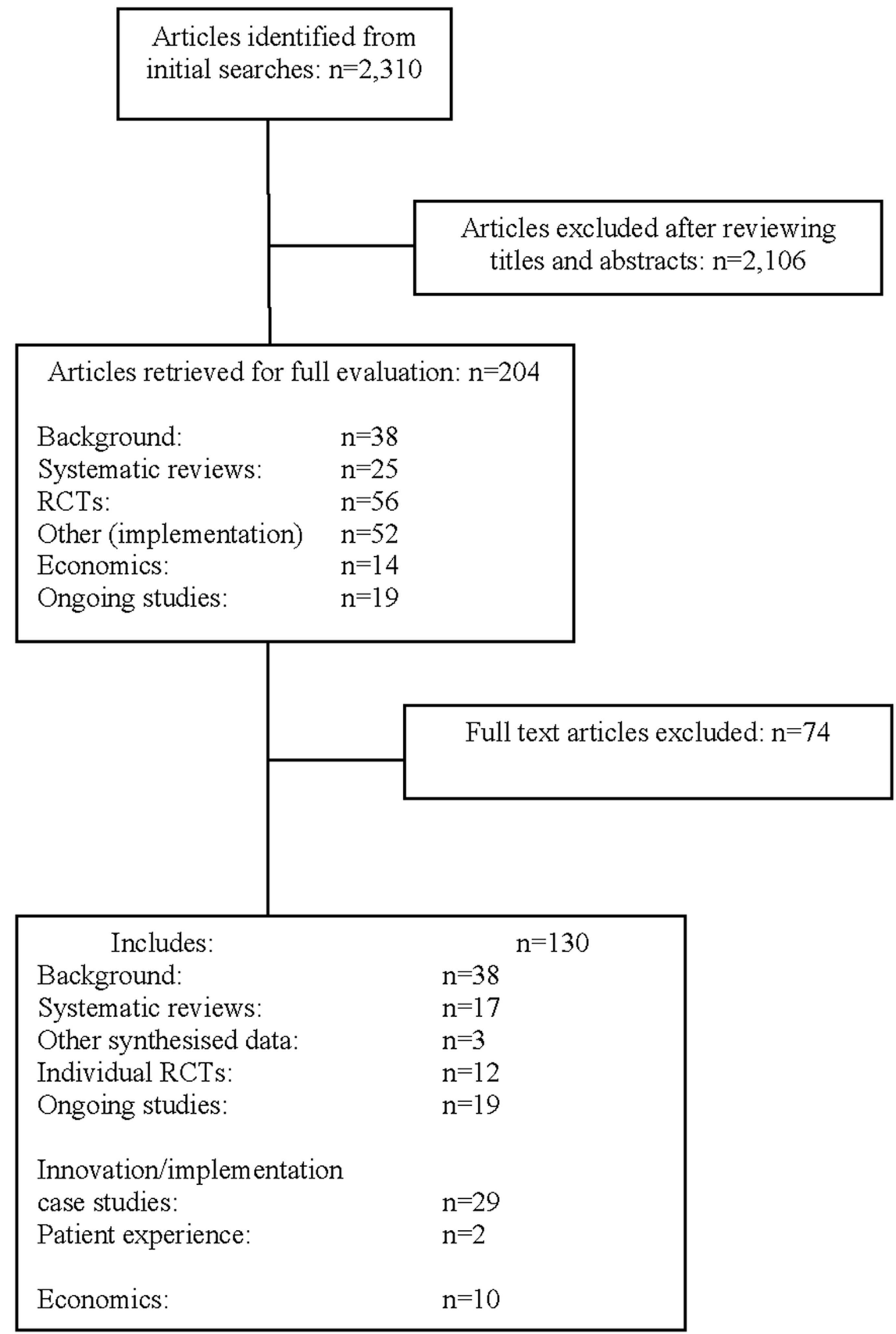

similar age ranges. Follow-up was generally up to 30 days post discharge.

The number and combination of ERAS elements varied considerably across all types of evidence; ranging from 4 to 14 elements across systematic reviews and from 10 to 14 elements across individual RCTs (see full report for details; in press). This highlights the lack of standardisation across ERAS programmes and agreement on what constitutes an ERAS pathway, and will have implications on the overall findings. Only one review assessed compliance with ERAS elements. ${ }^{7}$ Ahmed et $a l^{7}$ noted that, in general, compliance fell during the postoperative period in most of the studies (from around $100 \%$ to around $20 \%$ ). Use of epidural analgesia had the highest levels of compliance across all studies (67$100 \%)$. Use of transverse incisions had the lowest levels of compliance (around 25\%). Reasons for differences in compliance and waning of compliance were not measured in the reviews. None of the reviews assessed patient compliance, including adherence to preoperative advice to ensure fitness for surgery.

Despite the large number of studies, robust evidence was sparse (see online supplementary tables S1 and S2; full outcome details are available in the full review; in press). Seven reviews in colorectal surgery performed meta-analyses and showed a significant mean reduction in primary or total length of stay that ranged from 1.56 days (95\% CI 0.50 to 2.61 days) ${ }^{19}$ to 3.75 days $(95 \%$ 
Table 1 Systematic review risk of bias assessment

\begin{tabular}{|c|c|c|c|c|c|c|c|}
\hline Author & $\begin{array}{l}\text { Adequate } \\
\text { search }\end{array}$ & $\begin{array}{l}\text { Risk of } \\
\text { bias } \\
\text { assessed }\end{array}$ & $\begin{array}{l}\text { Quality } \\
\text { score } \\
\text { accounted } \\
\text { for in } \\
\text { analysis }\end{array}$ & $\begin{array}{l}\text { Study details } \\
\text { reported and } \\
\text { differences } \\
\text { accounted } \\
\text { for }\end{array}$ & $\begin{array}{l}\text { Statistical } \\
\text { heterogeneity } \\
\text { investigated }\end{array}$ & $\begin{array}{l}\text { Gaps in } \\
\text { research } \\
\text { identified }\end{array}$ & $\begin{array}{l}\text { Conclusions } \\
\text { justified }\end{array}$ \\
\hline \multicolumn{8}{|c|}{ Colorectal/colon surgery } \\
\hline Adamina et $a \wp$ & $\checkmark$ & $\checkmark$ & UC & $\checkmark$ & UC & $\checkmark$ & 2 \\
\hline Ahmed et al & $\checkmark$ & $\mathrm{x}$ & $\mathrm{x}$ & $x$ & $\mathrm{x}$ & $\mathrm{x}$ & $\checkmark$ \\
\hline Eskicioglu et a $f^{10}$ & $\checkmark$ & 2 & $x$ & $\checkmark$ & $\checkmark$ & $d$ & 2 \\
\hline Gouvas et $a l^{11}$ & $\checkmark$ & $\checkmark$ & $x$ & $\checkmark$ & 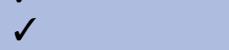 & $\checkmark$ & 4 \\
\hline Khan et $a l^{13}$ & $\checkmark$ & $\checkmark$ & $x$ & $d$ & $x$ & 2 & 2 \\
\hline Lv et $a P^{1}$ & $\checkmark$ & $\checkmark$ & $x$ & $x$ & $\checkmark$ & $\checkmark$ & $\checkmark$ \\
\hline Rawlinson et al ${ }^{15}$ & $\checkmark$ & $x$ & $x$ & $\checkmark$ & UC & $x$ & UC \\
\hline $\begin{array}{l}\text { Spanjersberg } \\
\text { et a } a^{16}\end{array}$ & $\checkmark$ & $\checkmark$ & $\checkmark$ & $\checkmark$ & $\checkmark$ & $\checkmark$ & $\checkmark$ \\
\hline Varadhan et al $1^{17}$ & $\checkmark$ & $\checkmark$ & $\mathrm{x}$ & $\checkmark$ & $\checkmark$ & $\checkmark$ & $\checkmark$ \\
\hline Walter et $a l^{18}$ & 2 & 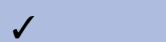 & $\checkmark$ & $\checkmark$ & 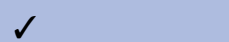 & 1 & 2 \\
\hline Wind et $a l^{19}$ & $\checkmark$ & $\checkmark$ & $\checkmark$ & $\checkmark$ & $\checkmark$ & $\checkmark$ & $\checkmark$ \\
\hline \multicolumn{8}{|c|}{ Gynaecological surgery } \\
\hline Lv et afo & 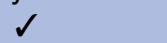 & $x$ & $\mathrm{x}$ & $\mathrm{x}$ & $\mathrm{x}$ & $\checkmark$ & 2 \\
\hline \multicolumn{8}{|c|}{ Liver/pancreatic surgery } \\
\hline Coolsen et $a^{\beta}$ & $\checkmark$ & $\checkmark$ & $\mathrm{x}$ & $\checkmark$ & $\mathrm{x}$ & $\checkmark$ & $\checkmark$ \\
\hline $\begin{array}{l}\text { Coolsen et a } \\
\text { Link to } 66\end{array}$ & $\checkmark$ & $\checkmark$ & $\checkmark$ & $\checkmark$ & $\checkmark$ & $\checkmark$ & $\checkmark$ \\
\hline Hall et al ${ }^{12}$ & $\mathrm{x}$ & $\mathrm{x}$ & $\mathrm{X}$ & $\checkmark$ & $\mathrm{x}$ & $\checkmark$ & $\checkmark$ \\
\hline \multicolumn{8}{|c|}{ Various surgical specialities } \\
\hline Lemmens et $a l^{14}$ & $\checkmark$ & $\mathrm{x}$ & $\mathrm{x}$ & $\checkmark$ & $x$ & $\checkmark$ & 2 \\
\hline Sturm et $a \bar{P}$ & 2 & $\mathrm{x}$ & $\mathrm{x}$ & 2 & UC & 2 & 2 \\
\hline
\end{tabular}

CI 5.11 to 2.40 days). ${ }^{18}$ Evidence from individual RCTs in colorectal surgery also suggest reduced length of hostpital stay following an ERAS programme (mean length of stay 4.15 to 6.43 days) compared to conventional care (mean length of stay 6.6 to 11.7 days). There were no significant differences in reported readmission rates, but it was unclear how readmissions were defined and measured in the reviews and RCTs.

Other surgical specialties showed greater variation in reported reductions in length of stay, but this is likely to reflect the greater uncertainty due to the more limited evidence base for these specialties. Statistical heterogeneity varied between reviews and was often not formally explored, but may have reflected differences in ERAS protocols, lack of compliance with important ERAS elements, and differences in surgical populations and procedures.

Deaths were rare and no significant differences between treatment groups were found in the systematic reviews and additional RCTs, regardless of surgical specialty. Morbidity was defined differently across systematic reviews and RCTs; rates between treatment groups were sometimes inconsistent, but generally indicated no statistically significant differences.

Mobilisation rates were inconsistent across systematic reviews, but most reported no significant differences in time to mobilisation between treatment groups. Mobilisation was rarely reported as an outcome in the additional RCTs.
Where systematic reviews and additional RCTs assessed quality of life and patient experience/satisfaction, equivocal findings were reported. Evidence on reintervention rates, pain and resource use was lacking in systematic reviews and RCTs.

\section{Other reviews}

A systematic review in colorectal surgery, identified after the last literature search, showed similar findings to the systematic reviews discussed above. ${ }^{36}$ Mean length of primary hospital stay was statistically significantly reduced in ERAS patients; mean difference (MD) -2.44 (95\% CI -3.06 to -1.83 ; 11 RCTs) but with significant statistical heterogeneity $\left(\mathrm{I}^{2}=88 \%\right)$. There was no evidence to suggest increased rates of readmissions, complications and mortality. Some of the individual RCT results for primary length of stay did not appear to be consistent with results reported in other systematic reviews, and this may have impacted on the estimated reduction in length of primary hospital stay. ${ }^{36}$

Two reviews ${ }^{37} 38$ focusing on individual ERAS elements were identified, of which highlighted the lack of evidence on the full ERAS pathway and the lack of compliance with ERAS protocols. Details can be found in the full review (in press). 


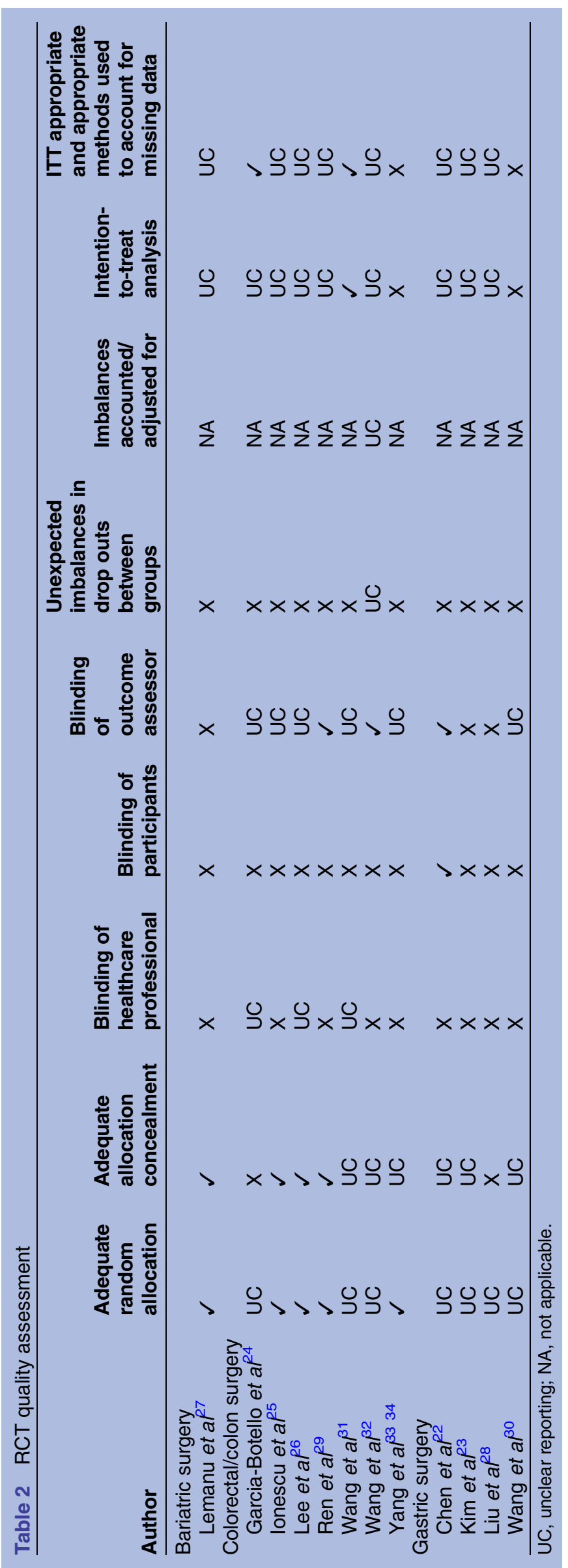

Case studies

Ten of 14 UK NHS innovation sites provided adequate data for inclusion in this section. ${ }^{39-41}$ Fifteen case studies of implementation of ERAS in NHS settings, and 11 NHS trusts (mostly in colorectal surgery) provided evidence relating to the implementation of an ERAS programme within their Trust. Full results and evidence tables are presented in the full review (in press).

There were variations in practice in terms of numbers and combinations of ERAS elements implemented; the most frequently implemented programme elements in the case studies were preadmission information/counselling and early postoperative mobilisation. Available evidence did not address which enhanced recovery elements and combinations of elements were most effective. Substantial variation in what constitutes an enhanced recovery programme within and between different surgical specialities, and difficulties in implementing certain ERAS components, suggest that the enhanced recovery pathway may be used as a framework and adapted to suit local situations. Evidence on compliance/adherence to enhanced recovery programmes was lacking.

Case studies identified the factors believed to act as barriers or facilitators to implementing an ERAS programme. Barriers to implementation included resistance to change from patients and staff, lack of funding or support from management, ${ }^{39}{ }^{42-44}$ staff turnover, problems arising from poor documentation, the time required to complete documentation and other practical issues.

Facilitators included the presence of a dedicated ERAS project lead/nurse to coordinate and sustain multidisciplinary working and continuity of the pathway, a multidisciplinary team approach and continual education for staff and patients/patient representatives. One innovation site mentioned that it did not offer a 7 day service for enhanced recovery due to staff resources. Patients operated on towards the end of the week may have to wait until after the weekend to be discharged if they need to be seen by any healthcare professionals or social services. The need to sustain multidisciplinary working means that, in the absence of $24 / 7$ working for elective procedures, enhanced recovery programmes will tend to be front loaded into the start of the working week (typically Monday to Thursday). Recent evidence suggests a higher risk of death for patients who have elective surgical procedures carried out later in the working week and at the weekend, ${ }^{45}$ the capcity to implement ERAS throughout the working week might ensure continuity of best care and help mitigate against such variation.

We included two published studies of patient experience of ERAS. ${ }^{46}{ }^{47}$ Each study used qualitative research methods to analyse audiotaped material. The two studies provided limited evidence suggesting that patients who were willing to provide feedback took a positive view of their experience of treatment in an ERAS programme. 
The studies suggested that patients were willing to comment on their experience in a way that can help healthcare providers to identify areas for improvement.

\section{COST-EFFECTIVENESS}

Ten economic evaluations in adult populations undergoing various surgical procedures evaluated costs and outcomes over short-time horizons (see online supplementary table S3). ${ }^{48-57}$ All of the evaluations suggested that programmes that achieve a reduction in length of stay are cost saving, and are not to the detriment of patients in terms of complication rates, readmission and health-related quality-of-life. The quality of the clinical studies on which these evaluations were based was variable, but generally poor. The generalisability of the results of these evaluations was limited by a lack of transparency in reporting, and the disparity in standard protocols and what had been evaluated across the settings made it unfeasible to select a cost-effective programme.

\section{DISCUSSION}

\section{Statement of principal findings}

Overall, the systematic reviews and additional RCTs suggest that length of hospital stay is reduced in ERAS patients compared to patients receiving conventional care. The evidence was based mainly on colorectal surgery and the applicability of findings to other surgical specialities remains less clear. Evidence for colorectal surgery suggests that enhanced recovery programmes may reduce hospital stays by $0.5-3.5$ days compared with conventional care.

There were marked differences in length of stay across reviews and individual studies regardless of specialty. These differences may reflect differences in ERAS protocols, compliance to ERAS programmes, healthcare systems and procedures and/or outcome definitions. This raises questions regarding the magnitude of effect of the ERAS protocols on length of stay, which may be overstated in some reviews.

The evidence suggests that ERAS programmes do not compromise patient morbidity, mortality and readmission rates but outcome definitions varied across reviews and individual studies. Such differences make it difficult to determine the reliability and generalisability of the findings.

Equivocal findings were reported for quality of life and patient experience/satisfaction but the evidence was based on few studies, which utilised various methods to measure these outcomes. The limited evidence precludes conclusions on the effects of ERAS protocols on pain, mobilisation and reintervention.

The implementation evidence included resource use in terms of the professionals involved in delivery of enhanced recovery programmes, but details were very limited and did not add to the evidence synthesis. Most case studies were uncontrolled and represent experiences of a sample of centres that chose to report their data; their outcomes may not be representative of those achieved elsewhere in the UK NHS. Their main value as evidence is the light they shed on NHS clinicians' perceptions of requirements for successful implementation and barriers to implementation of ERAS.

The impact of surgical experience and surgical volume on clinical outcomes was not explored and any implications of differences in these areas remain unknown. As enhanced recovery invariably targets the fitter, more mobile patient, frailer patients may not receive parity of access to what may be considered optimal treatment and management. Managers and clinicians considering implementing such programmes should think about the likely implication on equity of access. Whether inequity is an unintended outcome of enhanced recovery, merits further investigation.

Our review of the cost-effectiveness literature suggests that enhanced recovery programmes that achieve a reduction in length of stay may save costs without detrimental effects on complication rates, readmission and health-related quality of life. However, generalisability of the results of the economic evaluations is limited by a lack of transparency in reporting, use of different settings and populations and variable methodology in analyses. Data were lacking for resource use associated with the programmes evaluated and could not usefully inform the review of economic evaluations. In addition, the clinical effectiveness of some of the programmes considered in economic evaluations was not based on robust evidence.

\section{Strengths and weaknesses}

The main strength of this study was our use of multiple approaches to acquire and synthesise evidence. The main limitations were poor methodological quality and poor reporting of the included studies, and the inherent difficulty of reviewing a complex intervention in different healthcare systems and surgical specialities. Current methods for synthesising such complex interventions are limited. The methodological limitations and are not discussed here as this was outside the scope of this project, but have been addressed in previous publications. ${ }^{58}$ Another complication is that elements of early enhanced recovery programmes have become accepted practice within conventional care. This evolution makes combining studies over different periods and interpreting results of earlier studies in relation to the current context more difficult.

We found a large number of systematic reviews but there was substantial overlap in the included studies and evidence was not as abundant as the existence of multiple systematic reviews suggested. Most of the RCTs were small and not high quality. With the exception of one RCT, the remainder were single centre trials and therefore appear to have been undertaken to support implementation of an enhanced recovery programme in a specific setting rather than being planned as research studies. There were significant clinical and methodological differences between individual trials, and we therefore presented a 
narrative synthesis. Relatively few trials were conducted in the UK and this may limit the generalisability of evidence to UK NHS settings.

Lack of evidence on important outcomes including pain and quality of life is also an issue for research in this field. Trials tended not to report on adherence to the planned enhanced recovery programme. Assessing adherence to interventions and the impact this has on health outcomes is an important issue which is often overlooked in studies, and is a limitation in the evidence base in this review.

Three additional systematic reviews of effectiveness were brought to our attention during manuscript submission. One systematic review incorporates RCTs in colorectal surgery (Greco, 2013), ${ }^{59}$ one incorporates RCTs and cohort studies in abdominal surgery (Neville, $2014)^{60}$ and one includes RCTs and quasi-RCTs across various surgical specialities (Nicholson, 2014). ${ }^{61}$ The trials included in Greco $(2013)^{59}$ and Nicholson $(2014)^{61}$ overlap with those included in this review and the findings are consistent. The inclusion of these two reviews would therefore not have significantly altered the findings from this review. Neville $(2014)^{60}$ provides some additional data on patient-reported outcomes, including some evidence on post-discharge functional status. However, these outcomes were not frequently reported, and the additional evidence was mainly from study designs that would not have met the inclusion criteria for this review.

An important feature of our review is the inclusion of evidence on the implementation of enhanced recovery programmes in the UK NHS. This evidence has not been synthesised previously and the original programme websites are archived, so future access is not assured. By summarising this evidence, we have ensured that the main findings continue to be publicly available. We sought evidence on the experience of health professionals and patients of a broad range of sources and study types. Important themes emerged from this evidence that may be of value for implementing and sustaining enhanced recovery programmes in UK NHS settings. Owing to the rapid nature of the evidence synthesis, the list of sources searched to identify data on implementation and delivery of enhanced recovery programmes was not exhaustive and we acknowledge that relevant evidence may have been missed. Indeed, evidence from Scotland has been noted and eligible case studies have been identified from the NHS Scotland Quality Improvement Hub website. It should be noted that these are as limited as those included in the review. A qualitative study was brought to our attention at peer review; the study was published after our final search date. Pearsall et $a l^{62}$ conducted a qualitative study to explore the barriers and enablers in implementing an enhanced recovery after surgery programme in a University hospital in Canada. The themes identified are consistent with those reported in this review.
However, case studies are susceptible to risk of bias. Use of a standard reporting format was a potential strength of the case studies but variation in what each site actually reported (particularly in terms of evidence of benefit from the introduction of enhanced recovery programmes) reduced the usefulness of the evidence.

We sought to incorporate published and unpublished evidence on patient experiences and views of enhanced recovery programmes. Evaluation of patient experience of care is increasingly important for the NHS, especially in view of unacceptable failures of care such as those highlighted in the Francis Report. ${ }^{63}$ Though the evidence was generally positive for enhanced recovery, it was limited by a shortage of studies that used validated measures of patient experience and by study designs that could bias results in favour of enhanced recovery.

A further strength of this study was the consideration of cost-effectiveness evidence, but the nature of the evidence did not permit any analyses. There is a clear need to capture better evaluated data on costs and benefits of enhanced recovery programmes from a clearly stated perspective. A systematic review of economic evaluations ${ }^{64}$ was brought to our attention during manuscript publication. The review confirmed the need for welldesigned research to determine the cost-effectiveness of enhanced recovery programmes from the institutional and societal perspectives.

\section{Implications for healthcare}

Overall, there is consistent, albeit limited, evidence that enhanced recovery programmes can reduce length of patient hospital stay without increasing readmission rates. Data on reintervention rates and patient-reported outcomes did not suggest significant differences between enhanced recovery and conventional care, but the evidence was very limited and based on small numbers of patients. The lack of evidence on patient outcomes, resource use and costs precludes firm conclusions on the overall value of enhanced recovery programmes.

ERAS does not appear to reduce complication or readmission rates; the only cost benefit may lie in a reduction in post-operative bed days. Optimal care is certainly the right thing to do, but the evidence does not identify which enhanced recovery programme elements and combinations of elements are most effective. As such, conclusions on which combinations provide greatest gains and how best to implement them cannot be made.

The extent to which managers and clinicians considering implementing enhanced recovery programmes can realise reductions and cost savings will therefore depend on length of stays achieved under their existing care pathway. Important themes emerged from the relevant evidence identified on implementation, including the role of ERAS facilitators and the need for full support from management. It appears that these components are essential for the successful implementation and 
sustained delivery of enhanced recovery programmes in NHS settings. Consideration of potential benefit also needs to take account of the costs of service redesign, the resource use associated with programmes of this nature, the potential for improvement in patient outcomes and the impact on equity of access.

\section{Implications for research}

RCTs comparing an enhanced recovery programme with conventional care continue to be conducted and published, although mostly not in the UK. Given the available evidence, further single centre RCTs of this kind are not a priority. Rather, what is needed is improved collection and reporting of how enhanced recovery programmes are implemented, resourced and experienced in NHS settings. Also, exploration into the effect that varying levels of surgical volume and surgical experience and different discharge protocols might have on the success of an enhanced recovery pathway and subsequent outcomes.This will enhance our existing knowledge and understanding and provide evidence to support local decision-making about whether to adopt and how best to implement.

The two groups of implementation case studies included in our synthesis, although all were conducted in the UK, provide very limited information on how enhanced recovery programmes have actually been implemented in UK NHS settings. The standard reporting format originally proposed by The Enhanced Recovery Partnership Programme would enhance the value of future case studies if adhered to. Knowledge of how well the intervention has been implemented (fidelity) is essential for understanding how and why the intervention works and hence how outcomes can be further improved. Assessing fidelity may involve considering not only adherence to the requirements of the programme but also potential moderating factors, such as strategies used to assist delivery of the intervention, quality of delivery and participant responsiveness to new practices. ${ }^{65}$ It would be helpful if future innovation programmes used standardised reporting. For multisite programmes, a formal synthesis of findings from all participating sites should be undertaken as part of the evaluative process. This would ensure that the insights and contextual information which can inform the wider spread and adoption (or indeed discontinuation) would be systematically captured in a generalisable format.

Adherence/compliance to elements by staff and patients also requires further investigation. Rigorous data on patients' experiences of enhanced recovery programmes are lacking. Validated tools should be used and administered independently of those providing the service. Efforts should be made to obtain data from representative samples of patients receiving conventional care as well as those treated with enhanced recovery protocols, along with evidence on the experiences of their families/carers.
Evidence relating to the cost-effectiveness of enhanced recovery programmes in UK NHS settings is lacking. While enhanced recovery programmes have the potential to deliver cost savings, improved measurement of costs and benefits is crucial to help decisionmakers decide how best to make optimal use of limited resources.

Acknowledgements The authors would like to thank all the NHS staff that took the time to respond and help us identify grey literature considered for inclusion in this review.

Contributors PW took overall responsibility for the rapid synthesis. AE provided input at all stages. DC was involved in all stages of the economic evaluation including production of the final review write-up. DCh and FP were involved in all stages of the rapid synthesis including production of the final review write-up. DF conducted literature searches and contributed to the methods section of the review. DJ and EMcG provided advice throughout the rapid synthesis and commented on the draft review.

Funding This project was funded as part of a programme of research funded by the NIHR Health Services and Delivery Research programme (Project ref: 11/1026/04).

Disclaimer This report presents independent research funded by the National Institute for Health Research (NIHR). The views expressed are those of the authors and do not necessarily reflect those of the NIHR Health Services and Delivery Research programme or the Department of Health.

\section{Competing interests}

Provenance and peer review Not commissioned; externally peer reviewed.

Data sharing statement No additional data are available.

Open Access This is an Open Access article distributed in accordance with the Creative Commons Attribution Non Commercial (CC BY-NC 3.0) license, which permits others to distribute, remix, adapt, build upon this work noncommercially, and license their derivative works on different terms, provided the original work is properly cited and the use is non-commercial. See: http:// creativecommons.org/licenses/by-nc/3.0/

\section{REFERENCES}

1. Øvretveit J. Does improving quality save money? A review of evidence of which improvements to quality reduce costs for health service providers. London: The Health Foundation, 2009.

2. Øvretveit J. Does improving care coordination save money: a review of research. London: The Health Foundation, 2011.

3. Kehlet $\mathrm{H}$, Slim K. The future of fast-track surgery. Br J Surg 2012;99:1025-6.

4. Enhanced Recovery Partnership Programme. Delivering enhanced recovery-helping patients to get better sooner after surgery. London: Department of Health, 2010.

5. Sturm L, Cameron AL. Brief review: fast-track surgery and enhanced recovery after surgery (ERAS) programs. Melbourne: Australian Safety and Efficacy Register of New Interventional ProceduresSurgical (ASERNIP-S), 2009 Contract No:3.

6. Adamina M, Kehlet $H$, Tomlinson GA, et al. Enhanced recovery pathways optimize health outcomes and resource utilization: a meta-analysis of randomized controlled trials in colorectal surgery. Surgery 2011;149:830-40.

7. Ahmed J, Khan S, Lim M, et al. Enhanced recovery after surgery protocols-compliance and variations in practice during routine colorectal surgery. Colorectal Dis 2012;14:1045-51.

8. Coolsen MM, Wong-Lun-Hing EM, van Dam RM, et al. A systematic review of outcomes in patients undergoing liver surgery in an enhanced recovery after surgery pathways. HPB (Oxford) 2013;15:245-51.

9. Coolsen MM, van Dam RM, van der Wilt AA, et al. Systematic review and meta-analysis of enhanced recovery after pancreatic surgery with particular emphasis on pancreaticoduodenectomies. World J Surg 2013;37:1909-18.

10. Eskicioglu C, Forbes SS, Aarts M-A, et al. Enhanced recovery after surgery (ERAS) programs for patients having colorectal surgery: 
a meta-analysis of randomized trials. J Gastrointest Surg 2009;13:2321-9.

11. Gouvas N, Tan E, Windsor A, et al. Fast-track vs standard care in colorectal surgery: a meta-analysis update. Int $J$ Colorectal Dis 2009;24:1119-31.

12. Hall TC, Dennison AR, Bilku DK, et al. Enhanced recovery programmes in hepatobiliary and pancreatic surgery: a systematic review. Ann $R$ Coll Surg Engl 2012;94:318-26.

13. Khan S, Wilson T, Ahmed J, et al. Quality of life and patient satisfaction with enhanced recovery protocols. Colorectal Dis 2010;12:1175-82.

14. Lemmens L, van Zelm R, Borel Rinkes I, et al. Clinical and organizational content of clinical pathways for digestive surgery: a systematic review. Dig Surg 2009;26:91-9.

15. Rawlinson A, Kang $P$, Evans J, et al. A systematic review of enhanced recovery protocols in colorectal surgery. Ann $R$ Coll Surg Engl 2011:93:583-8.

16. Spanjersberg Willem R, Reurings J, Keus F, et al. Fast track surgery versus conventional recovery strategies for colorectal surgery. Cochrane Database of Sys Rev 2011;16(2):CD007635.

17. Varadhan KK, Neal KR, Dejong $\mathrm{CH}$, et al. The enhanced recovery after surgery (ERAS) pathway for patients undergoing major elective open colorectal surgery: a meta-analysis of randomized controlled trials. Clin Nutr 2010;29:434-40.

18. Walter CJ, Collin J, Dumville JC, et al. Enhanced recovery in colorectal resections: a systematic review and meta-analysis. Colorectal Dis 2009;11:344-53.

19. Wind J, Polle SW, Fung Kon Jin PH, et al. Systematic review of enhanced recovery programmes in colonic surgery. Br J Surg 2006;93:800-9.

20. Lu D, Wang $X$, Shi $G$. Perioperative enhanced recovery programmes for gynaecological cancer patients. Cochrane Database Syst Rev 2012;12:CD008239b.

21. Lv L, Shao YF, Zhou YB. The enhanced recovery after surgery (ERAS) pathway for patients undergoing colorectal surgery: an update of meta-analysis of randomized controlled trials. Int $J$ Colorectal Dis 2012;27:1549-54.

22. Chen Hu J, Xin Jiang L, Cai L, et al. Preliminary experience of fast-track surgery combined with laparoscopy-assisted radical distal gastrectomy for gastric cancer. J Gastrointest Surg 2012;16:1830-9.

23. Kim JW, Kim WS, Cheong JH, et al. Safety and efficacy of fast-track surgery in laparoscopic distal gastrectomy for gastric cancer: a randomized clinical trial. World J Surg 2012;36:2879-87.

24. Garcia-Botello S, Canovas de Lucas R, Tornero C, et al. Implementation of a perioperative multimodal rehabilitation protoco in elective colorectal surgery. A prospective randomised controlled study. Cir Esp 2011;89:159-66.

25. Ionescu D, lancu C, lon D, et al. Implementing fast-track protocol for colorectal surgery: a prospective randomized clinical trial. World $\mathrm{J}$ Surg 2009;33:2433-8.

26. Lee TG, Kang SB, Kim DW, et al. Comparison of early mobilization and diet rehabilitation program with conventional care after laparoscopic colon surgery: a prospective randomized controlled trial. Dis Colon Rectum 2011;54:21-8.

27. Lemanu DP, Singh PP, Berridge K, et al. Randomized clinical trial of enhanced recovery versus standard care after laparoscopic sleeve gastrectomy. Br J Surg 2013;100:482-9.

28. Liu XX, Jiang ZW, Wang ZM, et al. Multimodal optimization of surgical care shows beneficial outcome in gastrectomy surgery. JPEN J Parenter Enteral Nutr 2010;34:313-21.

29. Ren L, Zhu D, Wei Y, et al. Enhanced recovery after surgery (ERAS program attenuates stress and accelerates recovery in patients after radical resection for colorectal cancer: a prospective randomized controlled trial. World J Surg 2012;36:407-14.

30. Wang D, Kong Y, Zhong B, et al. Fast-track surgery improves postoperative recovery in patients with gastric cancer: a randomized comparison with conventional postoperative care. J Gastrointest Surg 2010;14:620-7.

31. Wang G, Jiang ZW, Xu J, et al. Fast-track rehabilitation program vs conventional care after colorectal resection: a randomized clinical trial. World J Gastroenterol 2011;17:671-6.

32. Wang Q, Suo J, Jiang J, et al. Effectiveness of fast-track rehabilitation vs conventional care in laparoscopic colorectal resection for elderly patients: a randomized trial. Colorectal Dis 2012;14:1009-13.

33. Yang DJ, Zhang S, He WL, et al. Fast track surgery accelerates the recovery of postoperative insulin sensitivity. Chin Med $J$ 2012;125:3261-5.

34. Yang DJ, Zhang S, He WL, et al. Fast-track surgery accelerates the recovery of postoperative humoral immune function in elective operation for colorectal carcinoma: a randomized controlled clinical trial. Chin Med J 2012;92:1112-15.

35. Vlug MS, Wind J, Hollmann MW, et al. Laparoscopy in combination with fast track multimodal management is the best perioperative strategy in patients undergoing colonic surgery: a randomized clinical trial (LAFA-study). Ann Surg 2011;254:868-75.

36. Cheng-Le Z, Xing-Zhao Y, Xiao-Dong Z, et al. Enhanced recovery after surgery programs versus traditional care for colorectal surgery: a meta-analysis of randomized controlled trials. Dis Colon Rectum 2013;56:667-78.

37. Arsalani-Zadeh R, Elfadl D, Yassin N, et al. Evidence-based review of enhancing postoperative recovery after breast surgery. $\mathrm{Br} J$ Surg 2011;98:181-96.

38. Hoffmann $\mathrm{H}$, Kettelhack $\mathrm{C}$. Fast-track surgery-conditions and challenges in postsurgical treatment: a review of elements of translational research in enhanced recovery after surgery. Eur Surg Res 2012;49:24-34.

39. Enhanced Recovery Partnership Programme Case Studies 2011 Gynaecology: Addenbrookes Hospital.

40. Enhanced Recovery Partnership Programme Case Studies 2011: Enhanced Recovery Programme: Yeovil District Hospital NHS Foundation Trust.

41. Enhanced Recovery Partnership Programme Case Studies 2011 Enhanced recovery for colorectal surgery: Yeovil District Hospital NHS Foundation Trust.

42. Enhanced Recovery Partnership Programme Case Studies 2011: Colorectal, Gynaecology, Urology, MSK: Medway NHS Foundation Trust.

43. Enhanced Recovery Partnership Programme Case Studies 2011: Colorectal surgery (all elective procedures) and most major emergencies from decision to treat surgically: Royal Berkshire Hospital.

44. Enhanced Recovery Partnership Programme Case Studies 2011: Enhanced recovery after colorectal surgery: Royal Berkshire Hospital.

45. Aylin $\mathrm{P}$, Alexandrescu $\mathrm{R}$, Jen $\mathrm{MH}$, et al. Day of week of procedure and 30 day mortality for elective surgery: retrospective analysis of hospital episode statistics. BMJ 2013;346:8

46. Blazeby JM, Soulsby M, Winstone $\mathrm{K}$, et al. A qualitative evaluation of patients' experiences of an enhanced recovery programme for colorectal cancer. Colorectal Dis 2010;12(10 Online) e236-42.

47. Taylor C, Burch J. Feedback on an enhanced recovery programme for colorectal surgery. Br J Nurs 2011;20:286-90.

48. Reilly KA, Beard DJ, Barker KL, et al. Efficacy of an accelerated recovery protocol for Oxford unicompartmental knee arthroplasty: a randomised controlled trial. Knee 2005;12:351-7.

49. Archibald LH, Ott MJ, Gale CM, et al. Enhanced recovery after colon surgery in a community hospital system. Dis Colon Rectum 2011:54:840-5.

50. Sammour T, Zargar-Shoshtari K, Bhat A, et al. A programme of enhanced recovery after surgery (ERAS) is a cost-effective intervention in elective colonic surgery. N Z Med J 2010;123:61-70.

51. King PM, Blazeby JM, Ewings $P$, et al. The influence of an enhanced recovery programme on clinical outcomes, costs and quality of life after surgery for colorectal cancer. Colorectal Dis 2006;8:506-13.

52. Nielsen PR, Andreasen J, Asmussen M, et al. Costs and quality of life for prehabilitation and early rehabilitation after surgery of the lumbar spine. BMC Health Serv Res 2008;8:209. http://www. biomedcentral.com/1472-6963/8/209

53. Jakobsen DH, Sonne E, Andreasen J, et al. Convalescence after colonic surgery with fast-track vs conventional care. Colorectal Dis 2006;8:683-7.

54. McBride N, Farringdon F, Midford R. Implementing a school drug education programme: reflections on fidelity. Int $\mathrm{J}$ Health Promot Educ 2002;40:40-50

55. Kariv Y, Delaney CP, Senagore AJ, et al. Clinical outcomes and cost analysis of a fast track postoperative care pathway for ileal pouch-anal anastomosis. A case control study. Dis Colon Rectum 2007:50:137-46.

56. Salhiyyah K, Elsobky S, Raja S, et al. A clinical and economic evaluation of fast-track recovery after cardiac surgery. Heart Surg Forum 2011;14:E330-4.

57. Yanatori M, Tomita S, Miura $\mathrm{Y}$, et al. Feasibility of the fast-track recovery program after cardiac surgery in Japan. Gen Thorac Cardiovasc Surg 2007;55:445-9.

58. Noyes J, Gough D, Lewin S, et al. A research and development agenda for systematic reviews that ask complex questions about complex interventions. J Clin Epidemiol 2013;66:1262-70. 
59. Greco M, Capretti G, Beretta L, et al. Enhanced Recovery Program in Colorectal Surgery: A Meta-analysis of Randomized Controlled Trials. World J Surg 2014;38:1531-41.

60. Neville A, Lee L, Antonescu I, et al. Systematic review of outcomes used to evaluate enhanced recovery after surgery. Br J Surg 2014;101:159-71.

61. Nicholson A, Lowe MC, Parker J, et al. Systematic review and metaanalysis of enhanced recovery programmes in surgical patients. $\mathrm{BrJ}$ Surg 2014;101:172-88.

62. Pearsall EA, Meghii Z, Pitzul KB, et al. A qualitative study to understand the barriers and enablers in implementing an enhanced recovery after surgery program. Ann Surg 18 Mar 2014. Epub ahead of print.
63. Report of the Mid Staffordshire NHS Foundation Trust Public Inquiry (Chaired by Robert Francis QC). London: The Stationery Office, 2013.

64. Lee L, Li C, Landry T, et al. A systematic review of economic evaluations of enhanced recovery pathways for colorectal surgery. Ann Surg 2014;259:670-6.

65. Carroll C, Patterson M, Wood S, et al. A conceptual framework for implementation fidelity. Implement Sci 2007;2:40.

66. Coolsen MME, van Dam RM, van der Wilt AA, et al. Systematic review and meta-analysis of enhanced recovery after pancreatic surgery with particular emphasis on pancreaticoduodenectomies: supplementary material. World J Surg 2013;37:1909-18. 